\title{
Novel Oral Anticoagulant versus Warfarin in Cancer Patients with Atrial Fibrillation: An 8-Year Population- Based Cohort Study
}

\author{
Victor Chien-Chia $\mathrm{Wu}^{1,2}$, Chun-Li Wang1,2, Yu-Tung Huang,3, Wen-Ching Lan ${ }^{3}$, Michael Wu ${ }^{5}$, Chang-Fu \\ $\mathrm{Kuo}^{2,6,7}$, Shao-Wei Chen ${ }^{8}$, Pao-Hsien Chu ${ }^{1,2}$, Ming-Shien Wen ${ }^{1,2}$, Chi-Ching Kuo ${ }^{9}$, Shang-Hung Chang ${ }^{1,2,3,4}{ }^{\bowtie}$ \\ 1. Division of Cardiology, Chang Gung Memorial Hospital, Linkou Medical Center, Taoyuan City, Taiwan \\ 2. College of Medicine, Chang Gung University, Taoyuan City, Taiwan \\ 3. Center for Big Data Analytics and Statistics, Chang Gung Memorial Hospital, Linkou Medical Center, Taoyuan City, Taiwan \\ 4. Graduate Institute of Nursing, Chang Gung University of Science and Technology, Taoyuan City, Taiwan \\ 5. Divison of Cardiovascular Medicine, Arrhythmia Services Section, Rhode Island Hospital, Warren Alpert School of Medicine, Brown University, \\ Providence, USA \\ 6. Division of Rheumatology, Allergy and Immunology, Department of Internal Medicine, Chang Gung Memorial Hospital, Linkou Medical Center, Taoyuan \\ City, Taiwan \\ 7. Division of Rheumatology, Orthopaedics and Dermatology, School of Medicine, University of Nottingham, Nottingham, UK \\ 8. Department of Cardiothoracic and Vascular Surgery, Chang Gung Memorial Hospital, Linkou Medical Center, Taoyuan City, Taiwan \\ 9. Institute of Organic and Polymeric Materials, National Taipei University of Technology, Taipei, Taiwan. \\ $\square$ Corresponding author: Shang-Hung Chang, MD, PhD, Division of Cardiology, Chang Gung Memorial Hospital, Linkou Medical Center, No. 5, Fuxing Street, \\ Guishan District, Taoyuan City 33305, Taiwan. Tel: +886-3-3281200 x8115; Fax: + 886-3-3281451; Email: afen.chang@gmail.com.
}

( $)$ The author(s). This is an open access article distributed under the terms of the Creative Commons Attribution License (https://creativecommons.org/licenses/by/4.0/). See http://ivyspring.com/terms for full terms and conditions.

Received: 2019.05.07; Accepted: 2019.09.20; Published: 2020.01.01

\begin{abstract}
Background: Cancer patients with atrial fibrillation (AF) were excluded in the major clinical trials. We therefore investigated the efficacy and safety of novel oral anticoagulant (NOAC) versus warfarin in these patients.

Methods: Data were retrieved from Taiwan National Health Insurance Research Database during 2010-2017 for patients with AF, excluding those without cancer or $>1$ cancer, not using anticoagulant, switching of agents, patients age $<18$, and cancer and AF diagnosed $>1$ month apart. Primary outcomes are ischemic stroke (IS)/systemic embolism (SE), Gl bleeding, major bleeding, intracranial hemorrhage (ICH), acute myocardial infarction (AMI), and death from any cause at 6 months and 1 year.

Results: After exclusion criteria and propensity score matching, there were 336 patients in each group. Patients on NOAC had significantly reduced $\mathrm{IS} / \mathrm{SE}(\mathrm{HR}=0.45,95 \% \mathrm{Cl}=0.25-0.82)$, major bleeding $(\mathrm{HR}=0.21,95 \% \mathrm{Cl}=0.05-0.96)$, and no $\mathrm{ICH}$ at 6 months. In addition, IS/SE (HR=0.42, 95\% $\mathrm{Cl}=0.24-0.74)$, major bleeding $(\mathrm{HR}=0.26,95 \% \mathrm{Cl}=0.09-0.76)$, and no $\mathrm{ICH}$ at 1 year compared to patients on warfarin. There was no difference on Gl bleeding, AMI, and death from any cause at 6 months and at 1 year.
\end{abstract}

Conclusion: In cancer patients with AF, NOAC were associated with significant reduced IS/SE, major bleeding, and $\mathrm{ICH}$ compared to warfarin.

Key words: anticoagulation, atrial fibrillation, cancer, NOAC, warfarin

\section{Introduction}

Patients with atrial fibrillation (AF) and a calculated $\mathrm{CHA}_{2} \mathrm{DS}_{2}$-VASc score $\geq 1$ in men and $\geq 2$ in women requires anticoagulation therapy with warfarin or novel oral anticoagulant (NOAC) [1]. Cancer is an important condition associated with development of $\mathrm{AF}[2,3]$. A higher cardiovascular risk 
factors has been noted in AF patients with cancer than without [4]. In cancer patients, there is increased risk of venous thromboembolism due to cancer-related disturbance of homeostasis and hypercoagulable state [5]. In addition, cancer patients undergoing chemotherapy frequently has complication of bleeding due to thrombocytopenia [6]. In cancer patients with AF, currently there is no guideline to direct our clinical management and they are less likely to receive adequate treatment for the concomitant diseases. Traditionally, the major landmark trials of NOAC with dabigatran, rivaroxaban, apixaban, and edoxaban for use in AF excluded patients with cancer [7-10]. Recently, edoxaban was found to be noninferior to subcutaneous low molecular weight heparin for the treatment of cancer-associated venous thromboembolism in the randomized clinical trial [11]. However, there is still lack of evidence supporting the use of NOAC over warfarin in these cancer patients with AF. Therefore, the aim of this study is to determine the efficacy and safety associated with use of NOAC versus warfarin in these patients.

\section{Materials and Methods}

\section{Data Source}

Taiwan's National Health Institute Program started in 1995 and provides $99.5 \%$ coverage for the 23 million residents in Taiwan. The NHI Research Database (NHIRD) provides all dates of inpatient and outpatient services, diagnoses, emergency room visits, prescriptions, examinations, operations, and expenditures, and data are updated biannually. With over $95 \%$ of Taiwanese population consists of Han Chinese, our study is considered of uniform ethnic background. The Institutional Review Board of Chang Gung Memorial Hospital Linkou Branch approved this study (IRB No. 201800725B1).

\section{Study Patients}

By searching electronic medical records from the NHIRD between January 1, 2010 and December 31, 2017, we retrieved patients with diagnosis of AF, using at least 1 inpatient or 2 outpatient claims for nonvalvular AF [12]. Active cancer was defined as cancer receiving treatment or diagnosed within last 6 months from previous report [12] and by NICE guide in United Kingdom and a previously published report [13], therefore we did not enroll patients whose follow-up period $>6$ months. Patients with cancer were screened, with exclusion criteria of no cancer or more than one type of cancer, not using oral anticoagulant (OAC) of either warfarin, dabigatran, rivaroxaban, apixaban, or edoxaban, switching of OAC agents, and patient age $<18$. In addition we exclude patients with cancer and AF diagnoses $>1$ month apart and use of OAC and AF diagnosis $>1$ month apart. Primary outcomes defined as ischemic stroke (IS)/systemic embolism (SE), GI bleeding, major bleeding, intracranial hemorrhage (ICH), acute myocardial infarction (AMI), and death from any cause at 6 months and 1 year.

\section{Study Outcomes and follow up}

We selected efficacy outcome of IS/SE, AMI, and death from any cause, and safety outcomes of major bleeding, GI bleeding, and ICH at 6 months and 1 year. The major bleeding was defined according to principle or secondary diagnosis of hospitalization and emergency visit and any blood transfusion order, which included admission for any bleeding, required blood transfusion $>2 \mathrm{U}$, and life-threatening bleeding or vital organ hemorrhage, which included ICH. Death from any cause was defined by withdrawal from the NHI program [14].

Disease was detected using International Classification of Diseases, 9th Revision, Clinical Modification (ICD-9-CM) and ICD-10 codes. Covariates included gender, age, medical history of diabetes mellitus, hypertension, hyperlipidemia, heart failure, renal insufficiency, peptic ulcer disease, abnormal liver function, peripheral arterial disease, prior ischemic stroke or systemic thromboembolism, old myocardial infarction, bleeding history, alcohol history, medication, $\mathrm{CHA}_{2} \mathrm{DS}_{2}$-VASc score, and HAS-BLED score. The comorbidity was defined as having two outpatient diagnoses or one inpatient diagnosis in the previous year. Similarly, usage of medication was retrieved based on claim data within six month before and after the index date.

\section{Ascertainment of Diseases and Comorbidities}

Our study retrieved data from NHIRD, using ICD-9-CM and ICD-10 disease coding with diagnoses defined as discharge diagnosis or at least two consecutive clinic visits. The accuracy of the diagnosis of has been confirmed and validated in previous studies [15-17]. In addition, Catastrophic Illnesses Registry, which all confirmed cancer patients are required to be registered, enhances the accuracy and completeness of data retrieval of patients with malignancy. The positive predictive value (PPV) of all cancers are $93.64 \%$ and the selected cancers are: cervical cancer, PPV 81.73\%; colorectal cancer, 94.38\%; esophageal cancer, PPV 94.45\%; female breast cancer, PPV 91.86\%; gastric cancer 91.84\%; liver and intrahepatic ducts cancer, PPV 93.03\%; lung cancer, PPV 94.90\%; oral cavity, oropharyngeal, and hypopharyngeal cancer, PPV 94.40\%; prostate cancer, 93.29\%; pancreatic cancer, PPV 90.39\% [16]. In 
addition, the PPV of selected comorbidities are: diabetes, PPV 92\%; hypertension, PPV 88.5\%; hyperlipidemia, PPV 89.5\%; heart failure, PPV 97.6\%; myocardial infarction, PPV 92.0\%; ischemic stroke, PPV 97.9\% [17].

\section{Statistical Analysis}

Each patient who was in warfarin group was matched to a patient in the NOAC group. We compared the baseline characteristics, comorbidities, and medication, $\mathrm{CHA}_{2} \mathrm{DS}_{2}-\mathrm{VASc}$ score, and HAS-BLED score between the study groups using t-test for continuous variable or chi-square test for categorical variable. We compared the risk of death from any cause between groups using a Cox proportional hazard model. We generated the plot of cumulative probability using subdistribution hazard function for time to event outcomes. A $P$ value $<0.05$ was considered to be statistically significant. No adjustment of multiple testing (multiplicity) was made in this study. All statistical analyses were performed using commercial software (SAS 9.4, SAS Institute, Cary, NC).

\section{Sensitivity Analysis}

In order to validate our study findings and check for potential selection biases, we performed the sensitivity analysis. In contrast to the main analysis which we exclude patients with $\mathrm{AF}$ and cancer diagnosed $>1$ month apart, we re-analyzed the data with $\mathrm{AF}$ and cancer diagnosis $>1$ year, which is similar to ARISTOTLE study on apixaban that enrolled patients with cancer within the previous 12 months. The result of the main study was compared to different enrollment criteria to assess whether the primary findings would be modified.

\section{Results}

\section{Study Population}

There were 319,697 patients with a principal diagnosis of AF during 2010 and 2017 identified in the NHIRD. After exclusion criteria, there remained 933 patients with 477 on warfarin and 456 on NOAC eligible for analysis (Figure 1). After propensity score matching, there were 336 patients on warfarin and 336 patients on NOAC for study (Table 1). Before propensity score matching, patients who were on warfarin were younger, had lower prevalence of diabetes mellitus, hypertension, hyperlipidemia, abnormal liver function, peripheral artery disease, and alcoholic history. After propensity score matching there were no difference between the two groups. Before propensity score matching, CHA2DS-VASc score were $3.92 \pm 1.93$ in patients on warfarin, and $4.34 \pm 1.97$ in patients on NOAC. After propensity score matching, CHA2DS-VASc score were $4.20 \pm 1.89$ in patients on warfarin, and $4.21 \pm 2.00$ in patients on NOAC. Before propensity score matching, HAS-BLED score were $2.93 \pm 1.58$ in patients on warfarin, and $3.38 \pm 1.42$ in patients on NOAC. After propensity score matching, HAS-BLED score were $3.19 \pm 1.56$ in patients on warfarin, and $3.25 \pm 1.44$ in patients on NOAC.

\section{Patients on warfarin versus patients on NOAC}

Patients on NOAC had significantly reduced IS/SE (hazard ratio [HR] 0.45, 95\% confidence interval [CI] 0.25-0.82), major bleeding (HR 0.21, 95\% CI 0.05-0.96), and no ICH at 6 months, compared to patients on warfarin. In addition, patients on NOAC had significantly reduced IS/SE (HR $0.42,95 \%$ CI 0.24-0.74), major bleeding (HR 0.26, 95\% CI 0.09-0.76), and no ICH at 1 year, compared to patients on warfarin (Figure 2, Figure 3). There was no difference on GI bleeding, AMI, and death from any cause between the two groups at 6 months and at 1 year.

\section{Sensitivity Analysis}

With enrollment criteria change to exclude patients with diagnosis of $\mathrm{AF}$ and cancer $>1$ year apart, the results were similar for IS/SE, GI bleeding, ICH, AMI, and death from any cause at 6 months of follow up, and the results were similar for IS/SE, major bleeding, ICH, AMI, and death from any cause at 1 year (Supplementary Figure 1). The consistent findings were the outcomes of IS/SE, ICH, AMI, and death from any cause using enrollment criteria of patients with diagnosis of AF and cancer both within 1 month and within 1 year.

\section{Discussions}

To our knowledge, this is the first study to investigate the efficacy and safety of cancer patients with AF who were on warfarin or on NOAC in ethnic Chinese. Our study had two findings. (1) In cancer patients with $\mathrm{AF}$, patients on NOAC had significant reduced IS/SE, major bleeding, and ICH compared to patients on warfarin. (2) There was no difference on GI bleeding, AMI, and death from any cause between the two groups at 6 months and at 1 year.

$\mathrm{AF}$ has been found to develop in patients with cancer due to comorbid diseases or direct tumor effect. Given that both diseases occurred with increased incidence in aging population, the 2 conditions are found to be coexist with increased frequency in clinical scenarios [18]. Although major clinical trials of NOAC for stroke prevention in patients with AF reported benefits of reduced IS/SE and major bleeding compared use of warfarin, the trials excluded patients with cancer therefore there 
was the knowledge gap in the anticoagulation use in cancer patients with AF. Due to cancer related hypercoagulability, venous thromboembolism, and cancer-induced thrombocytopenia; it has been complicated to manage these patients with AF. As a matter of fact, it is possible that these patients were frequently undertreated.

A recent retrospective study compared the effectiveness of NOAC which included rivaroxaban, dabigatran, and apixaban to warfarin in patients with $\mathrm{AF}$ and cancer [12]. The authors reported no significant difference in IS in these 3 NOACs compared to warfarin, and no significant difference in severe bleeding in rivaroxaban and dabigatran to warfarin [12]. There was however significantly reduced severe bleeding in apixaban compared to warfarin $(p=0.01)$ [12]. In this study however, edoxban was not included in the comparison. Current evidence of comparative treatment efficacy and bleeding complication with edoxaban and warfarin was only available from the management of venous thromboembolism in Houkosai Study [11]. Although edoxaban was noninferior to subcutaneous dalteparin for recurrent venous thromboembolism, there was a significantly increased bleeding among edoxaban users [11]. Another recently published study investigated provider specialty, anticoagulation, and stroke risk in patients with $\mathrm{AF}$ and cancer, using anticoagulation prescription filled 3 months prior to and 6 months after AF diagnosis as enrollment criteria [19]. The author noted that patients with history of cancer were less likely to fill prescription with anticoagulation [19]. In addition, patients with cancer were more likely to fill prescription if seen by a cardiologist with reduced risk of stroke (HR 0.89, 95\% CI 0.81-0.99) and without an increased risk of bleeding (HR 1.04, 95\%, CI 0.95-1.13) [19].

Table 1. Clinical characteristics of study patients

\begin{tabular}{|c|c|c|c|c|c|c|}
\hline \multirow{2}{*}{ Variable } & \multicolumn{3}{|c|}{ Before matching } & \multicolumn{3}{|c|}{ After matching } \\
\hline & $\begin{array}{l}\text { On warfarin } \\
(\mathrm{n}=477)\end{array}$ & $\begin{array}{l}\text { On NOAC } \\
(\mathrm{n}=456)\end{array}$ & P value & $\begin{array}{l}\text { On warfarin } \\
(\mathrm{n}=336)\end{array}$ & $\begin{array}{l}\text { On NOAC } \\
(\mathrm{n}=336)\end{array}$ & $\mathrm{P}$ value \\
\hline \multicolumn{7}{|l|}{ Characteristic } \\
\hline Gender (male) & $293(61.43)$ & $287(62.94)$ & 0.6338 & $213(63.39)$ & $209(62.2)$ & 0.7495 \\
\hline Mean age & $73.62 \pm 10.41$ & $75.61 \pm 9.63$ & 0.0025 & $75.08 \pm 9.5$ & $75.09 \pm 9.9$ & 0.9910 \\
\hline Age group & & & 0.0107 & & & 0.6698 \\
\hline $18-64$ years. & $98(20.55)$ & $62(13.6)$ & & $48(14.29)$ & $55(16.37)$ & \\
\hline $65-74$ years & $140(29.35)$ & $131(28.73)$ & & $103(30.65)$ & 95 (28.27) & \\
\hline$\geq 75$ years & $239(50.1)$ & $263(57.68)$ & & $185(55.06)$ & $186(55.36)$ & \\
\hline \multicolumn{7}{|l|}{ Medical history } \\
\hline Diabetes mellitus & $163(34.17)$ & $194(42.54)$ & 0.0085 & $132(39.29)$ & $135(40.18)$ & 0.8130 \\
\hline Hypertension & $338(70.86)$ & $369(80.92)$ & 0.0003 & $256(76.19)$ & $265(78.87)$ & 0.4055 \\
\hline Hyperlipidemia & $158(33.12)$ & $225(49.34)$ & $<.0001$ & 139 (41.37) & $145(43.15)$ & 0.6394 \\
\hline Heart failure & $134(28.09)$ & $129(28.29)$ & 0.9466 & $99(29.46)$ & $97(28.87)$ & 0.8652 \\
\hline Renal insufficiency & $132(27.67)$ & $148(32.46)$ & 0.111 & $101(30.06)$ & $105(31.25)$ & 0.7379 \\
\hline Peptic ulcer disease & $164(34.38)$ & $185(40.57)$ & 0.0508 & $129(38.39)$ & $133(39.58)$ & 0.7517 \\
\hline Abnormal liver function & $80(16.77)$ & $100(21.93)$ & 0.0459 & $65(19.35)$ & $64(19.05)$ & 0.9220 \\
\hline Peripheral artery disease & $33(6.92)$ & $49(10.75)$ & 0.039 & $28(8.33)$ & $29(8.63)$ & 0.8899 \\
\hline Prior ischemic stroke or systemic thromboembolism & $85(17.82)$ & $74(16.23)$ & 0.5181 & $58(17.26)$ & $60(17.86)$ & 0.8393 \\
\hline Old myocardial infarction & $24(5.03)$ & $27(5.92)$ & 0.5502 & $17(5.06)$ & $18(5.36)$ & 0.8622 \\
\hline Alcoholic history & $5(1.05)$ & $13(2.85)$ & 0.0454 & $5(1.49)$ & $<5$ & $0.7249 \#$ \\
\hline \multicolumn{7}{|l|}{ Medication } \\
\hline Antiplatelets & $285(59.75)$ & $299(65.57)$ & 0.0662 & $214(63.69)$ & $226(67.26)$ & 0.3302 \\
\hline $\mathrm{ACEi} / \mathrm{ARB}$ & $302(63.31)$ & $289(63.38)$ & 0.9836 & $216(64.29)$ & $224(66.67)$ & 0.5163 \\
\hline Amiodarone/dronedarone & $139(29.14)$ & $90(19.74)$ & 0.0008 & $77(22.92)$ & $79(23.51)$ & 0.855 \\
\hline Beta blockers & $288(60.38)$ & $290(63.6)$ & 0.3114 & $203(60.42)$ & $218(64.88)$ & 0.2316 \\
\hline Calcium channel blockers & $258(54.09)$ & $284(62.28)$ & 0.0112 & $199(59.23)$ & $208(61.9)$ & 0.4775 \\
\hline Diuretics & $204(42.77)$ & $156(34.21)$ & 0.0073 & $137(40.77)$ & $135(40.18)$ & 0.8751 \\
\hline NSAIDs & $173(36.27)$ & $248(54.39)$ & $<.0001$ & $160(47.62)$ & $157(46.73)$ & 0.8167 \\
\hline Oral hypoglycemic agents & $153(32.08)$ & $166(36.4)$ & 0.1636 & $120(35.71)$ & $123(36.61)$ & 0.8097 \\
\hline $\mathrm{CHA}_{2} \mathrm{DS}_{2}$-VASc score & $3.92 \pm 1.93$ & $4.34 \pm 1.97$ & 0.0011 & $4.2 \pm 1.89$ & $4.21 \pm 2.0$ & 0.9369 \\
\hline $\mathrm{CHA}_{2} \mathrm{DS}_{2}$-VASc score group & & & 0.0048 & & & 0.2946 \\
\hline 2-3 (Moderate) & $153(32.08)$ & $110(24.12)$ & & $103(30.65)$ & $84(25)$ & \\
\hline 4-5 (High) & $157(32.91)$ & $176(38.6)$ & & $114(33.93)$ & $129(38.39)$ & \\
\hline$\geq 6$ (Very high) & $111(23.27)$ & $132(28.95)$ & & $92(27.38)$ & $89(26.49)$ & \\
\hline HAS-BLED score & $2.93 \pm 1.58$ & $3.38 \pm 1.42$ & $<.0001$ & $3.19 \pm 1.56$ & $3.25 \pm 1.44$ & 0.5899 \\
\hline HAS-BLED score group & & & $<.0001$ & & & 0.1544 \\
\hline $0-2$ (Low) & $185(38.78)$ & $113(24.78)$ & & $111(33.04)$ & $94(27.98)$ & \\
\hline$\geq 3$ (High) & $292(61.22)$ & $343(75.22)$ & & $225(66.96)$ & $242(72.02)$ & \\
\hline
\end{tabular}

$\mathrm{ACEi}$, angiotensin converting enzyme inhibitor; ARB, angiotensin receptor blocker; COPD, chronic obstructive pulmonary disease; non-steroidal anti-inflammatory drug. \#Fisher exact test

Numbers $<5$ are not shown, as per confidentiality policies of the Taiwan National Health Insurance Research Database. 
Patients with Diagnosis of

AF between

January 1, 2010 and December 31, 2017

$(\mathrm{N}=319,697)$

Screening for coexisting cancer and exclude:

1. No cancer or more than one type of cancer $(N=279,952)$

2. Not using OAC of either warfarin, dabigatran, rivaroxaban, apixaban or edoxaban ( $N=20,233$ )

3. Switching of OAC agents $(N=6,653)$

4. Patient $<18$ years old $(N=1)$

Patients with Diagnoses of

Cancer and AF

( $N=12,858)$

Exclude:

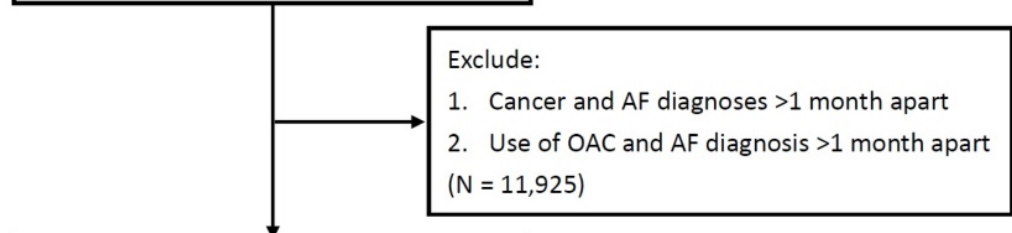

Patients with Diagnoses of

Cancer and AF under OAC

$$
(\mathrm{N}=933)
$$

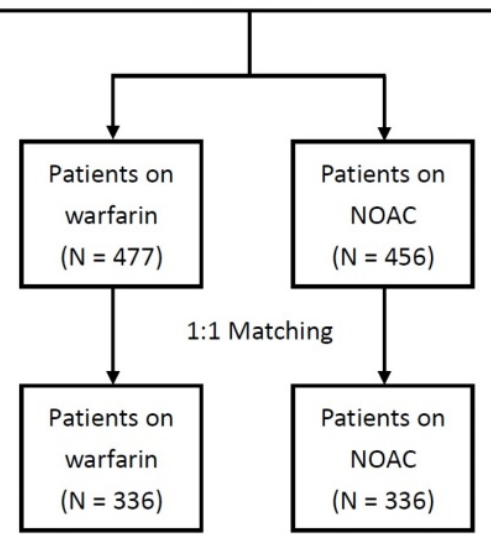

Figure 1. Study design and screening criteria flow chart for the inclusion of cancer patients with atrial fibrillation (AF). OAC, oral anticoagulant

6 months follow-up
IS/SE
Major bleeding*
GIB
ICH
AMI
Death
1 year follow-up
IS/SE
Major bleeding*
GIB
ICH
AMI
Death

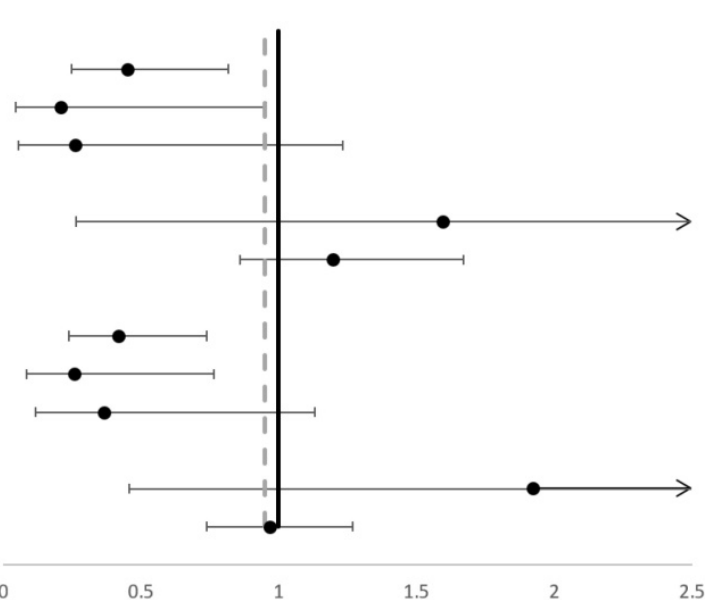

HR

0.452

0.209

0.262

0

1.596

1.198

0.42

0.257

0.365

0

1.923

0.969
$95 \% \mathrm{Cl}$

(0.25-0.817)

(0.046-0.956)

(0.056-1.233)

(-)

(0.266-9.555)

(0.86-1.67)

(0.239-0.739)

(0.086-0.764)

(0.118-1.131)

$(-)$

$(0.459-8.066)$

(0.739-1.27)

0

0.5

1.5

2.5

Figure 2. Primary outcomes occurred during 6 months and 1 year follow up. AMI, acute myocardial infarction; GIB, gastrointestinal bleeding; ICH, intracranial hemorrhage; IS/SE, ischemic stroke/systemic embolism. 
A

IS/SE

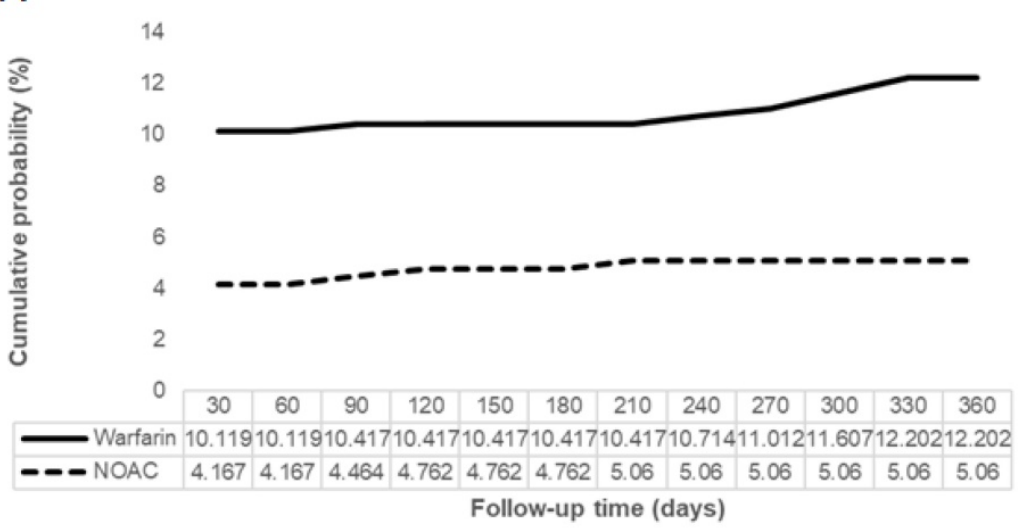

-Warfarin - - NOAC
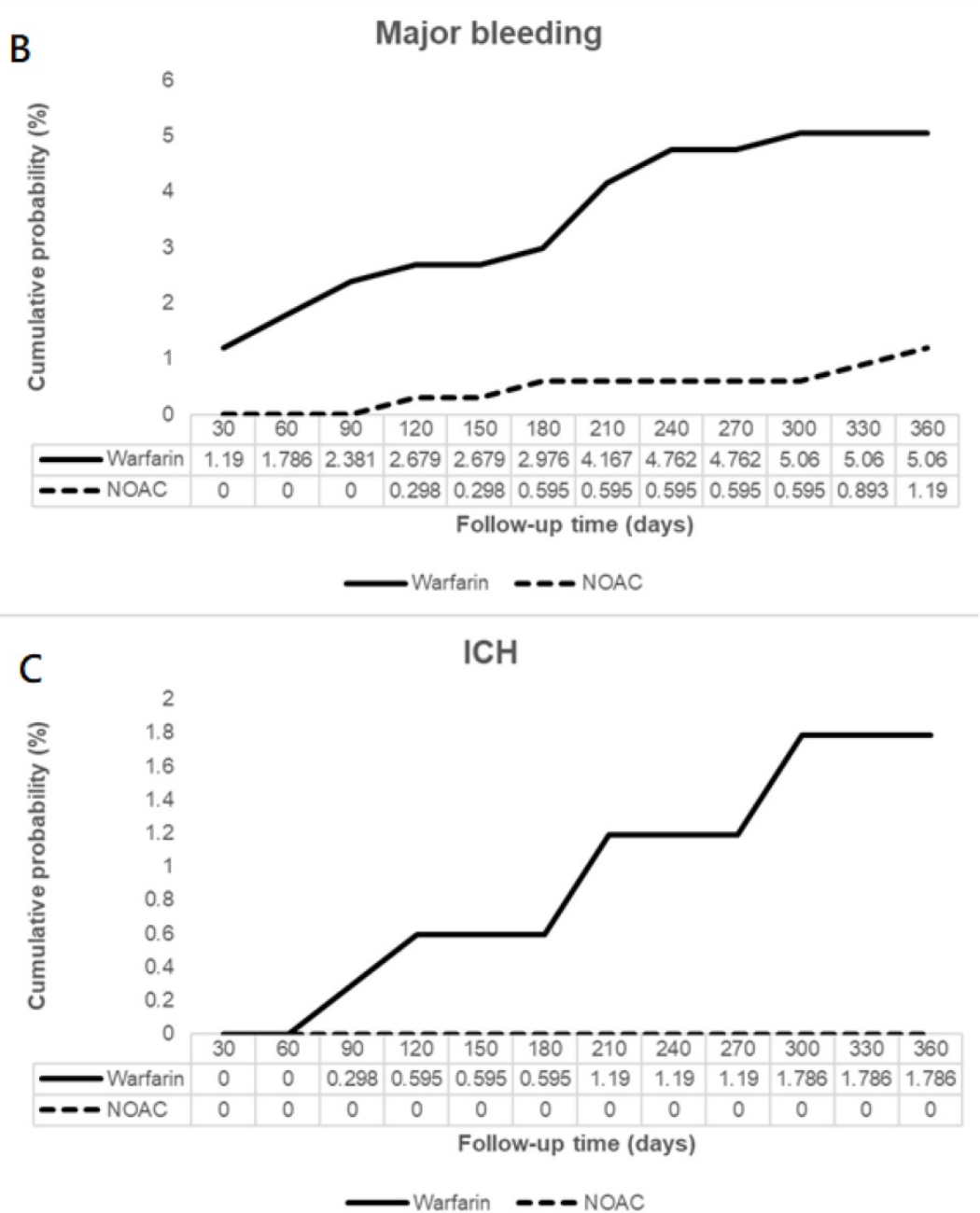

Figure 3. Cumulative probabilities of ischemic stroke (IS)/systemic embolism (SE) (A), major bleeding (B), and intracranial hemorrhage (ICH) (C).

In a recent retrospective study, efficacy and safety of NOACs among Korean patients with AF and newly diagnosed cancer were studied. In the study which propensity score was performed for age, sex, comorbidities, $\mathrm{CHA}_{2} \mathrm{DS}_{2}$-VASc and HAS-BLED scores, the authors reported the significantly decreased incidence of IS/SE, major bleeding, and all-cause mortality in NOACs treated group as compared to those with warfarin. In addition, these results were not affected by different type or reduced dosage of NOACs in up to $71.9 \%$ patients in the NOACs group [20]. 
In this study, we retrospectively enrolled patients with AF and then screened for coexisting cancer and excluded patients with more than one type of concomitant cancer. In contrast to previous study which did not exclude patients who switched between different anticoagulants [12], our study excluded patients who had switching of OAC agents. In addition, using stricter criteria compared to previous studies $[19,20]$, we excluded patients whose diagnoses of cancer and AF were $>1$ month apart, and use of $\mathrm{OAC}$ and $\mathrm{AF}$ diagnosis $>1$ month apart. After exclusion criteria, we then propensity score matched these patients, including age, sex, comorbidities, $\mathrm{CHA}_{2} \mathrm{DS}_{2}$-VASc and HAS-BLED scores, and additionally the co-administered medications, resulting 336 patients on warfarin and 336 patients on NOAC for analysis. During 6 months and 1 year follow up, we found patients on NOAC had significantly reduced IS/SE, major bleeding, and no incidence of ICH at 6 months, compared to patients on warfarin. The same results extended to 1 year with significantly reduced IS/SE, major bleeding, and still no incidence of ICH. There was no difference on GI bleeding, AMI, and death from any cause at 6 months and at 1 year. In summary, in comparison to warfarin, NOAC appears to be effective for prevention of ischemic stroke and systemic embolism in cancer patients with AF while no increased bleeding events occurred.

\section{Limitations}

There are several limitations in epidemiologic data from NHIRD. First, using ICD-9-CM and ICD-10 codes for patient screening may miss some cases for conditions not coded correctly. Second, due to the limitation of NHIRD where detailed report of imaging study on ischemic stroke and intracranial hemorrhage were not available therefore the extent of tissue damage could not be quantified, we could still obtain incidence from the diagnosis for the occurrence of ischemia or hemorrhage. Third, we did not assess the quality of warfarin control by calculating the time in therapeutic range, since these data are not available in the NHIRD. Fourth, we did not analyze individual NOACs to delineate the efficacy and safety of each drug compared to warfarin since the number of later induced edoxaban user would be small. Further studies to generalize our study results to other populations thus are warranted.

\section{Conclusions}

In cancer patients with AF, NOAC was associated with significant reduced ischemic stroke/systemic embolism, major bleeding, and intracranial hemorrhage compared to warfarin.
NOAC may be considered in preference to warfarin in these patients.

\section{Supplementary Material}

Supplementary figures.

http://www.jcancer.org/v11p0092s1.pdf

\section{Acknowledgments}

The authors thank the statistical assistance and wish to acknowledge the support of the Maintenance Project of the Center for Big Data Analytics and Statistics at Chang Gung Memorial Hospital for study design and monitor, data analysis and interpretation. In addition, Shang-Hung Chang and Chi-Ching Kuo acknowledge Chang Gung Memorial Hospital Joint Research Program and the Ministry of Science and Technology, Taiwan, and National Taipei University of Technology and for financial support. Center for Big Data Analytics and Statistics Grant CORPG3G0271 and CLRPG3D0045 at Chang Gung Memorial Hospital. Research Grant NTUT-CGMH108-07 at NTUT-CGMH Joint Research Program.

\section{Competing Interests}

The authors have declared that no competing interest exists.

\section{References}

1. Kirchhof P, Benussi S, Kotecha D, et al. 2016 ESC Guidelines for the management of atrial fibrillation developed in collaboration with EACTS. Eur Heart J. 2016;37:2893-962.

2. Guzzetti S, Costantino G, Vernocchi A, et al. First diagnosis of colorectal or breast cancer and prevalence of atrial fibrillation. Intern Emerg Med. 2008;3:227-31.

3. O'Neal WT, Lakoski SG, Qureshi W, et al. Relation between cancer and atrial fibrillation (from the REasons for Geographic And Racial Differences in Stroke Study). Am J Cardiol. 2015;115:1090-4.

4. Melloni C, Shrader P, Carver J, et al. Management and outcomes of patients with atrial fibrillation and a history of cancer: the ORBIT-AF registry. Eur Heart J Qual Care Clin Outcomes. 2017;3:192-7.

5. Timp JF, Braekkan SK, Versteeg HH, et al. Epidemiology of cancer-associated venous thrombosis. Blood. 2013;122:1712-23.

6. Liebman HA. Thrombocytopenia in cancer patients. Thromb Res. 2014;133 Suppl 2;S63-9.

7. Connolly SJ, Ezekowitz MD, Yusuf S, et al. Dabigatran versus warfarin in patients with atrial fibrillation. N Engl J Med. 2009;361:1139-51.

8. Patel MR, Mahaffey KW, Garg J, et al. Rivaroxaban versus warfarin in nonvalvular atrial fibrillation. N Engl J Med. 2011;365:883-91.

9. Granger $\mathrm{CB}$, Alexander JH, McMurray JJ, et al. Apixaban versus warfarin in patients with atrial fibrillation. N Engl J Med. 2011;365:981-92.

10. Giugliano RP, Ruff CT, Braunwald E, et al. Edoxaban versus warfarin in patients with atrial fibrillation. N Engl J Med. 2013;369:2093-104.

11. Raskob GE, van Es N, Verhamme P, et al. Edoxaban for the treatment of cancer-associated venous thromboembolism. N Engl J Med. 2018;378:615-24.

12. Shah S, Norby FL, Datta YH, et al. Comparative effectiveness of direct oral anticoagulants and warfarin in patients with cancer and atrial fibrillation. Blood Adv. 2018;2:200-9.

13. "Quality statement 7: Treatment of people with active cancer." Venous thromboembolism in adults: diagnosis and management, April 2016, www.nice.org.uk/guidance/qs29/chapter/quality-statement-7-treatment-ofpeople-with-active-cancer. Assessed September 9, 2017.

14. Wu CY, Chen YJ, Ho HJ, et al. Association between nucleoside analogues and risk of hepatitis $B$ virus-related hepatocellular carcinoma recurrence following liver resection. JAMA. 2012;308:1906-14.

15. Hung MC, Lai WW, Chen $\mathrm{HH}$, et al. Cost effectiveness of cancer treatment in Taiwan. J Formos Med Assoc. 2016;115:609-18.

16. Kao WH, Hong JH, See LC, et al. Validity of cancer diagnosis in the National Health Insurance database compared with the linked National Cancer Registry in Taiwan. Pharmacoepidemiol Drug Saf. 2018;27:1060-6. 
17. Hsieh CY, Su CC, Shao SC, et al. Taiwan's National Health Insurance Research Database: past and future. Clin Epidemiol. 2019;11:349-58.

18. Farmakis D, Parissis J, Filippatos G. Insights into onco-cardiology: atrial fibrillation in cancer. J Am Coll Cardiol. 2014;63:945-53.

19. O'Neal WT, Claxton JS, Sandesara PB, et al. Provider specialty, anticoagulation, and stroke risk in patients with atrial fibrillation and cancer. J Am Coll Cardiol. 2018;72:1913-22.

20. Kim K, Lee YJ, Kim TH, et al. Effect of non-vitamin K antagonist oral anticoagulants in atrial fibrillation patients with newly diagnosed cancer. Korean. Circ J 2018;48:406-17. 